\title{
Ankyrin-B in susceptibility to obesity
}

Overeating and lack of physical activity are the leading causes of obesity; however, genetics might also be involved. Strong evidence has linked many genetic loci to obesity, but the mechanism remains elusive. A new study has shown that dysfunction of ankyrin-B (a protein expressed by the proposed obesity susceptibility gene ANK2) in adipose tissue results in cell-autonomous adiposity by increasing glucose uptake and lipogenesis.

Previous studies by Damaris Lorenzo, Vann Bennett and colleagues found that ankyrin-B mutant mice consumed more glucose and exhibited increased levels of GLUT4 in adipose tissue and striated muscle. Mice deficient in ankyrin-B in only adipocytes showed the same results, suggesting that the observed effects were due to fat cells.

In their new study, Lorenzo and Bennett used in vitro binding assays using purified proteins and co-immunoprecipitation to demonstrate that ankyrin-B directly couples GLUT4 to clathrin to promote their association in adipocytes. Dysfunction of ankyrin-B resulted in inefficient GLUT4 return from the cell surface, which was associated with increased glucose uptake and lipogenesis. "Thus, we identified ankyrin-B as a novel clathrin adaptor in adipocytes," says Lorenzo.

Lorenzo and Bennett also identified seven human mutations in ankyrin-B that caused adiposity in an in vivo rescue assay in ankyrin-B-deficient adipocytes. These mutations are candidates for susceptibility to obesity in humans. "This work advances our knowledge of the role of the membrane-associated cytoskeleton in cellular and systemic metabolism, and metabolic disease," concludes Lorenzo. "The ankyrin-B variants characterized in this study are cumulatively found in over 6 million Americans of different ethnicities; therefore, dysfunction of ankyrin-B in adipocytes could have broad health effects."

Ivone Leong

ORIGINAL ARTICLE Lorenzo, D. N. \& Bennett, V.

Cell-autonomous adiposity through increased cell surface GLUT4 due to ankyrin-B deficiency. Proc. Natl Acad. Sci USA http://dx.doi.org/10.1073/ pnas.1708865114 (2017)

FURTHER READING Lorenzo, D. N. et al.

Ankyrin-B metabolic syndrome combines agedependent adiposity with pancreatic $B$ cell insufficiency.J. Clin. Invest. 125, 3087-3102 (2015) 